Supporting Information

\title{
Evaporated Perovskite Thick Junctions for X-ray Detection
}

Wei $\mathrm{Li}^{\dagger}$, Yalun $\mathrm{Xu}^{\dagger}$, Jiali Peng, Ruiming Li, Jiannan Song, Huihuang Huang, Lihao Cui, Qianqian Lin*

Key Laboratory of Artificial Micro- and Nano-Structures of Ministry of Education of China, School of Physics and Technology, Wuhan University, Wuhan 430072, P. R. China

†W.L. and Y.X. contributed equally.

\section{Corresponding Author:}

*Email: q.lin@whu.edu.cn 


\section{Supporting figures and tables}

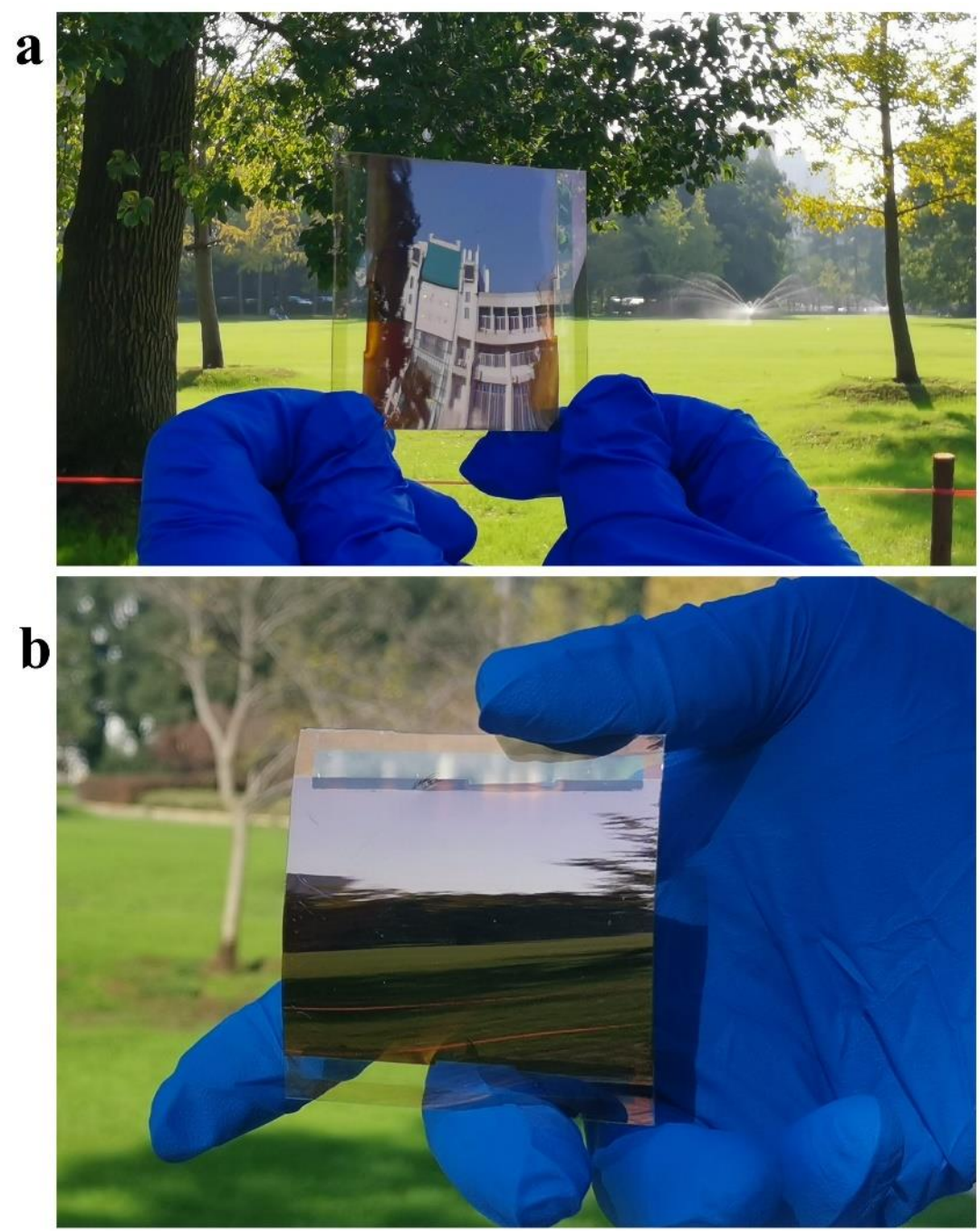

Figure S1. Photographs of the (a) front and (b) back of a large-area, evaporated perovskite films deposited on the flexible PET substrate. 
Table S1. The device performance statistics of perovskite photodiodes based on evaporated $\mathrm{FAPbI}_{3}$ film with various in-situ annealing temperatures. The parameters of the best device are shown in brackets.

\begin{tabular}{|c|c|c|c|c|}
\hline & $J_{\mathrm{SC}}\left(\mathbf{m A ~ ~ ^ { - 2 }}\right)$ & $V_{\text {OC }}(\mathbf{V})$ & FF & PCE $(\%)$ \\
\hline $125^{\circ} \mathrm{C}$ & $18.31 \pm 0.2(18.46)$ & $1.04 \pm 0.02(1.05)$ & $0.62 \pm 0.03(0.65)$ & $12.4 \pm 0.2(12.6)$ \\
\hline $\mathbf{1 3 0} \mathbf{C}$ & $\mathbf{2 3 . 5 6} \pm \mathbf{0 . 1 2}(\mathbf{2 3 . 6 8})$ & $\mathbf{0 . 9 8} \pm \mathbf{0 . 0 1}(\mathbf{0 . 9 9})$ & $\mathbf{0 . 7 6} \pm \mathbf{0 . 0 2}(\mathbf{0 . 7 8})$ & $\mathbf{1 7 . 9 \pm 0 . 2 ( 1 8 . 1 )}$ \\
\hline $135^{\circ} \mathrm{C}$ & $19.83 \pm 0.19(19.96)$ & $1.02 \pm 0.01(1.03)$ & $0.69 \pm 0.02(0.71)$ & $14.4 \pm 0.2(14.6)$ \\
\hline $140^{\circ} \mathrm{C}$ & $15.69 \pm 0.13(15.76)$ & $1.05 \pm 0.02(1.07)$ & $0.64 \pm 0.03(0.67)$ & $11.1 \pm 0.2(11.3)$ \\
\hline $145^{\circ} \mathrm{C}$ & $7.02 \pm 0.1(7.03)$ & $1.14 \pm 0.02(1.15)$ & $0.48 \pm 0.04(0.52)$ & $4.1 \pm 0.1(4.2)$ \\
\hline
\end{tabular}

Table S2. The device performance statistics of perovskite photodiodes based on evaporated $\mathrm{FAPbI}_{3}$ film with various hole transport layers. The parameters of the best device are shown in brackets.

\begin{tabular}{|c|c|c|c|c|}
\hline & $J_{\mathrm{SC}}\left(\mathbf{m A ~} \mathrm{cm}^{-2}\right)$ & $V_{\text {OC }}(\mathbf{V})$ & FF & PCE (\%) \\
\hline poly-TPD & $23.12 \pm 0.17(\mathbf{2 3 . 2 9})$ & $0.97 \pm 0.01(\mathbf{0 . 9 8})$ & $0.76 \pm 0.01(\mathbf{0 . 7 7})$ & $17.4 \pm 0.2(\mathbf{1 7 . 6})$ \\
\hline PTAA & $22.47 \pm 0.19(\mathbf{2 2 . 6 6})$ & $0.97 \pm 0.01(\mathbf{0 . 9 8})$ & $0.71 \pm 0.03(\mathbf{0 . 7 4})$ & $16.2 \pm 0.2(\mathbf{1 6 . 4})$ \\
\hline PEDOT:PSS & $19.49 \pm 0.21(\mathbf{1 9 . 7 0})$ & $0.95 \pm 0.02(\mathbf{0 . 9 7})$ & $0.62 \pm 0.02(\mathbf{0 . 6 4})$ & $12.0 \pm 0.2(\mathbf{1 2 . 2})$ \\
\hline $\mathrm{NiO}_{\mathrm{x}}$ & $18.87 \pm 0.19(\mathbf{1 9 . 0 6})$ & $0.96 \pm 0.01(\mathbf{0 . 9 7})$ & $0.59 \pm 0.02(\mathbf{0 . 6 1})$ & $11.0 \pm 0.3(\mathbf{1 1 . 3})$ \\
\hline TAPC & $9.19 \pm 0.18(\mathbf{9 . 3 7})$ & $0.86 \pm 0.02(\mathbf{0 . 8 8})$ & $0.27 \pm 0.03(\mathbf{0 . 2 9})$ & $2.2 \pm 0.2(\mathbf{2 . 4})$ \\
\hline $\mathrm{CuI}$ & $10.01 \pm 0.18(\mathbf{1 0 . 1 8})$ & $0.82 \pm 0.02(\mathbf{0 . 8 4})$ & $0.27 \pm 0.03(\mathbf{0 . 7 6})$ & $6.3 \pm 0.2(\mathbf{6 . 5})$ \\
\hline
\end{tabular}



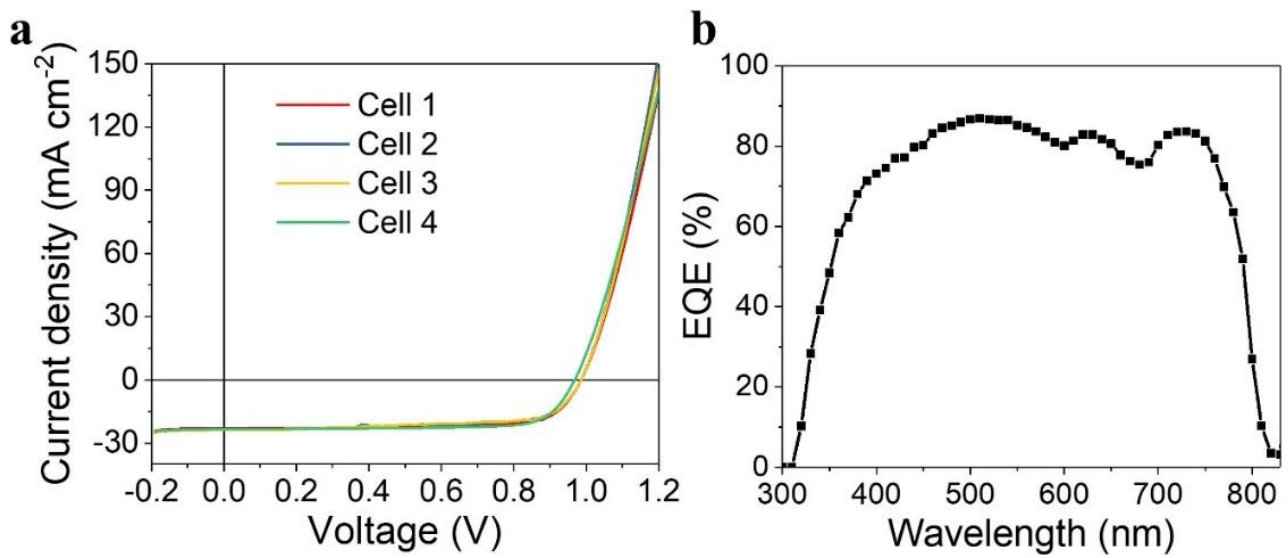

Figure S2. (a) The $J-V$ characteristics of multiple photodiodes and (b) typical EQE spectra of the optimized photodiode.

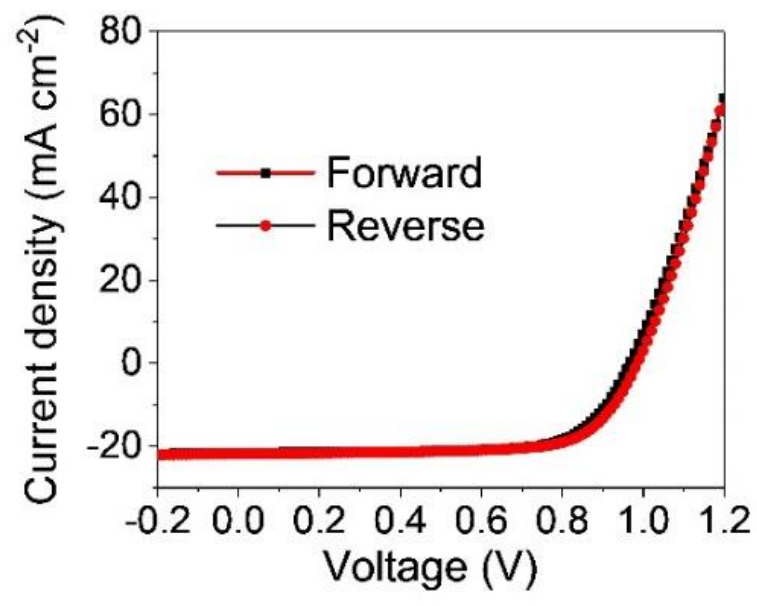

Figure S3. Typical $J$ - $V$ curves of the evaporated perovskite devices with forward and reverse scans under 1 sun illumination. 

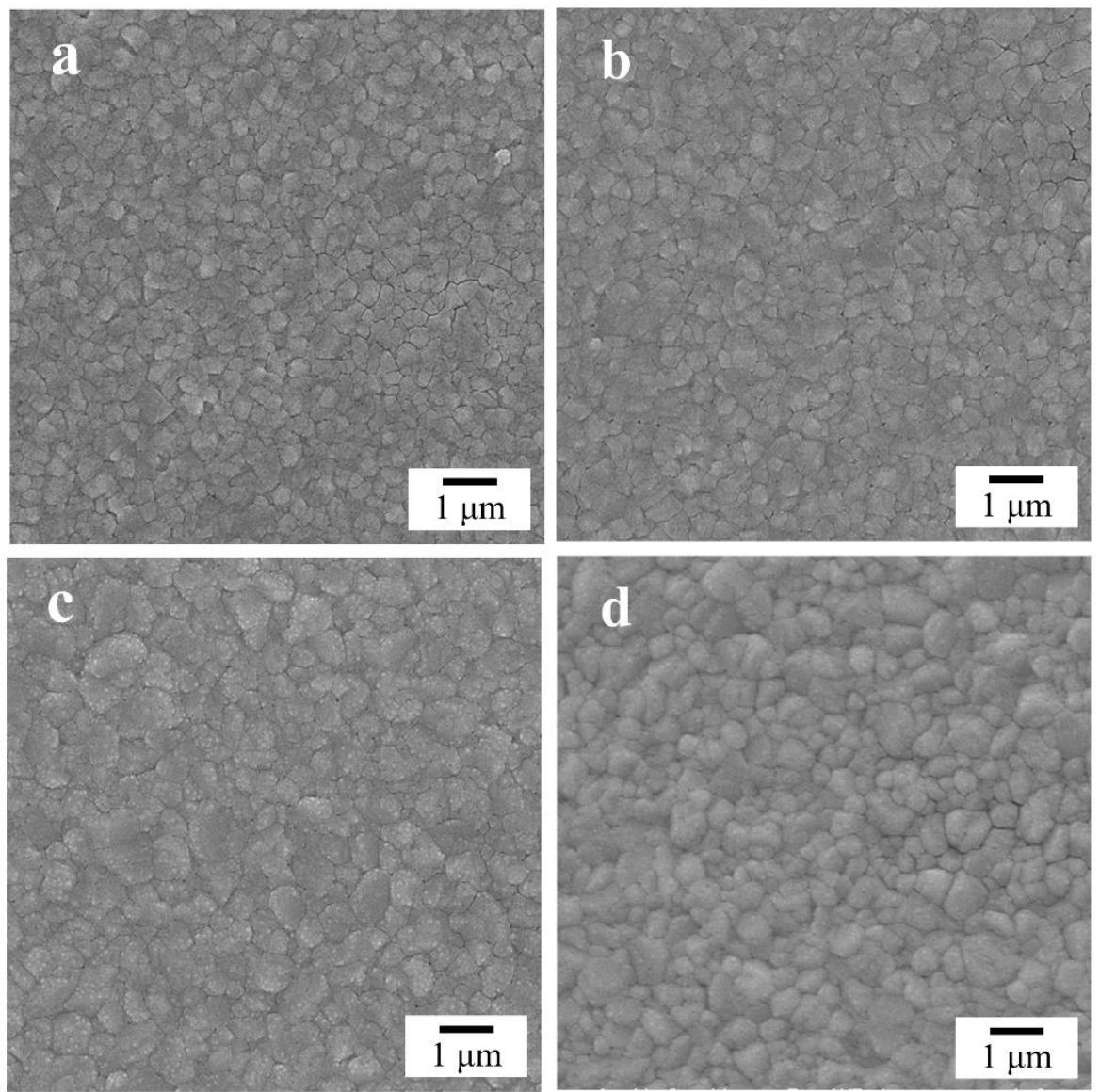

Figure S4. SEM images of (a) $0.7 \mu \mathrm{m}$, (b) $1.5 \mu \mathrm{m}$, (c) $3 \mu \mathrm{m}$ and (d) $5 \mu \mathrm{m}$ evaporated $\mathrm{FAPbI}_{3}$ perovskite films. 


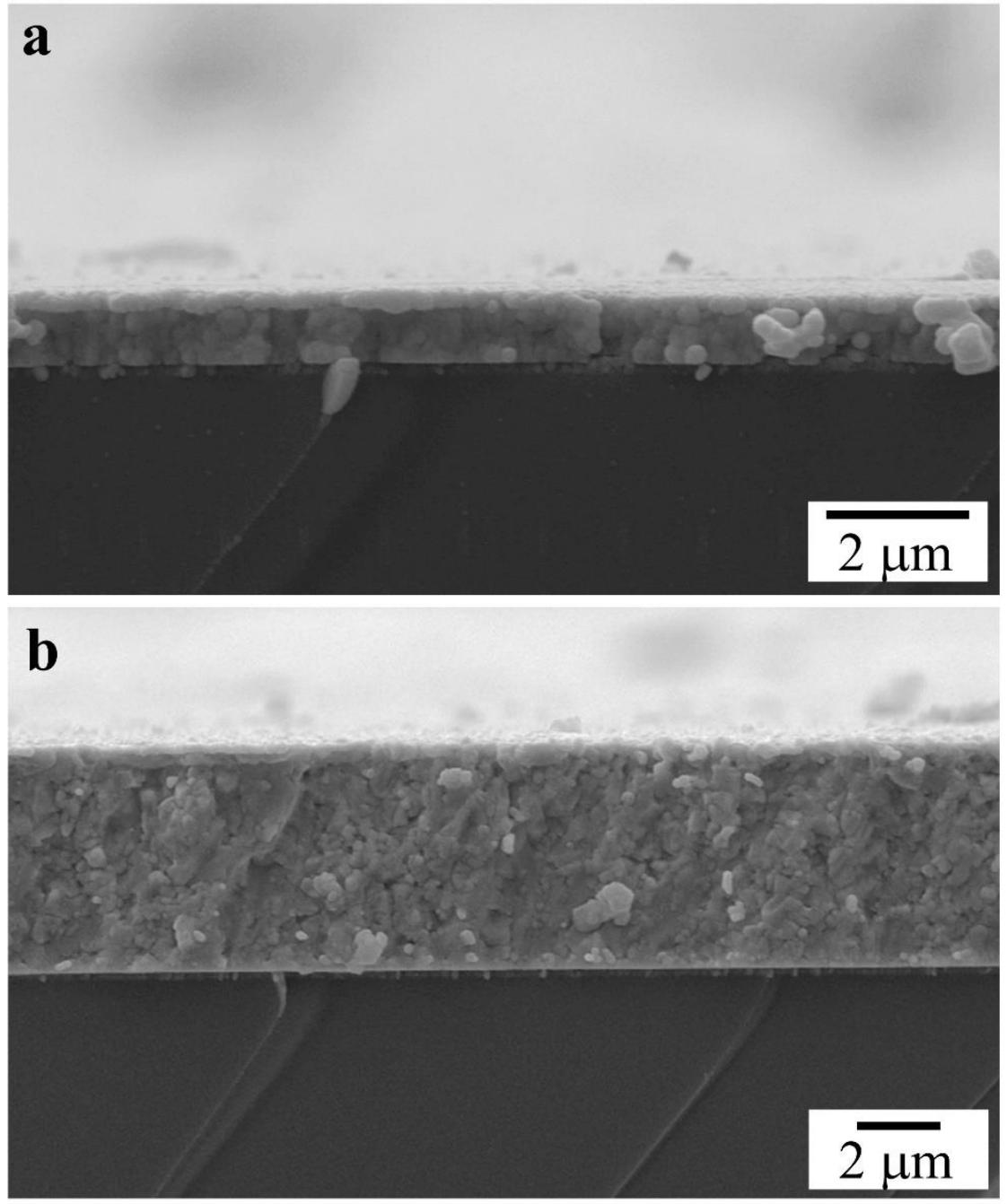

Figure S5. SEM images of cross-sectional view of the perovskite photodiodes with active layer thicknesses of (a) $0.7 \mu \mathrm{m}$ and (b) $5 \mu \mathrm{m}$. 


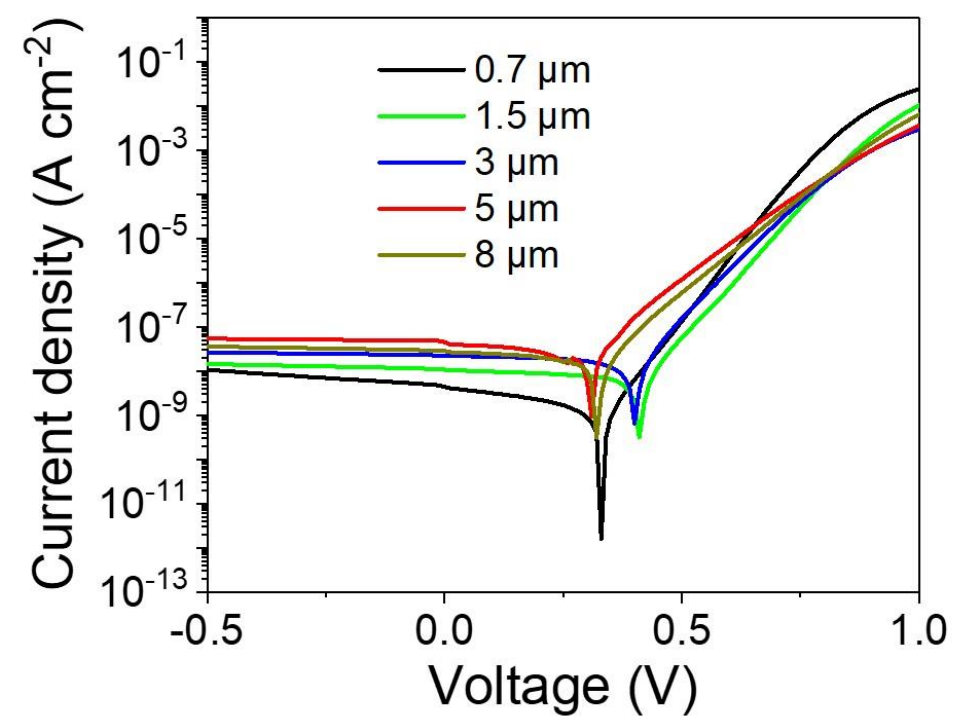

Figure S6. $J$ - $V$ characteristics of the photodiodes with various thicknesses under the same $\mathrm{X}$ ray dose rate of $412.9 \mu \mathrm{Gy}_{\text {air }} \mathrm{s}^{-1}$.

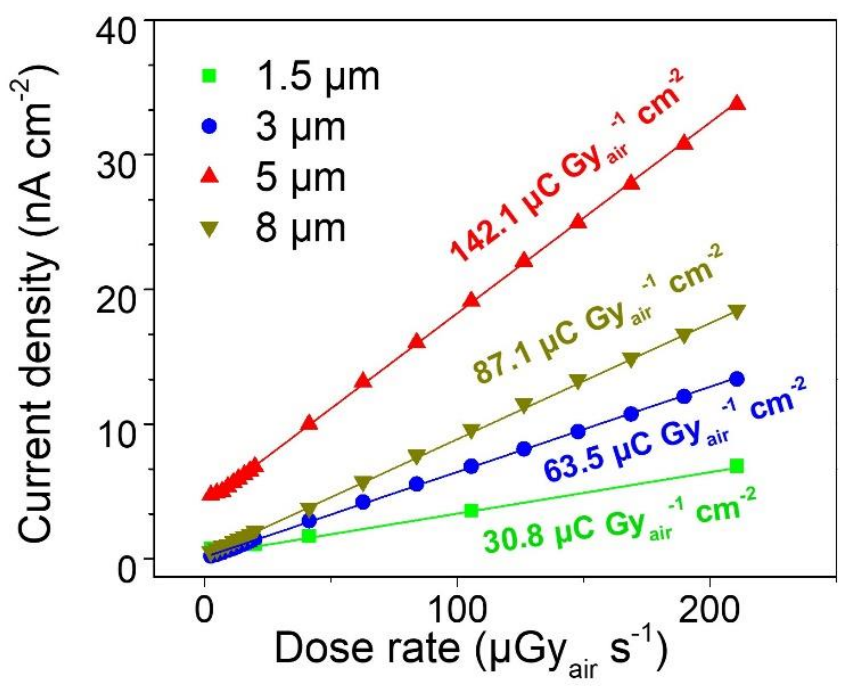

Figure S7. The comparison of the sensitivity of the evaporated perovskite X-ray detectors with various thicknesses at $-0.5 \mathrm{~V}$. 

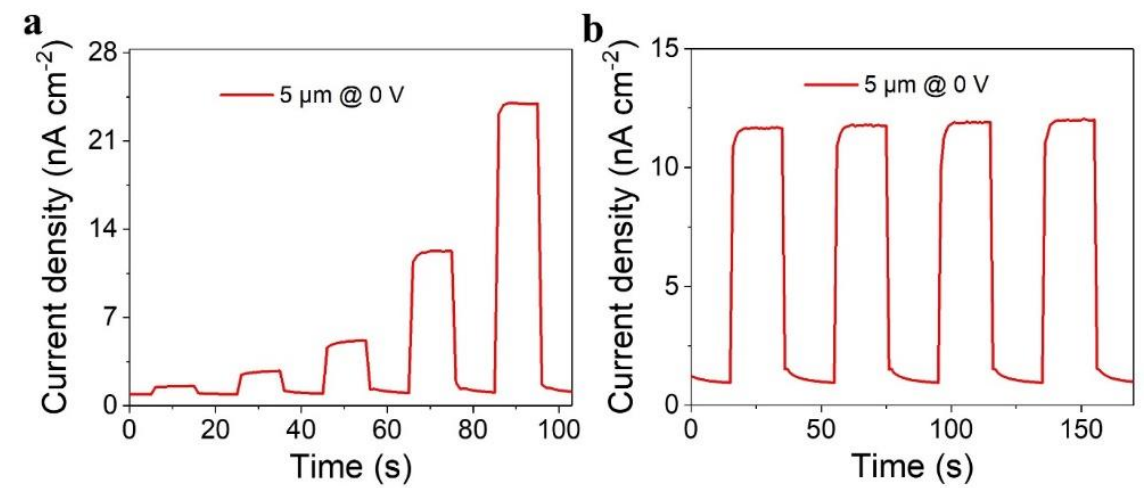

Figure S8. The temporal response of the optimized EPXDs with active layer thickness of $5 \mu \mathrm{m}$.

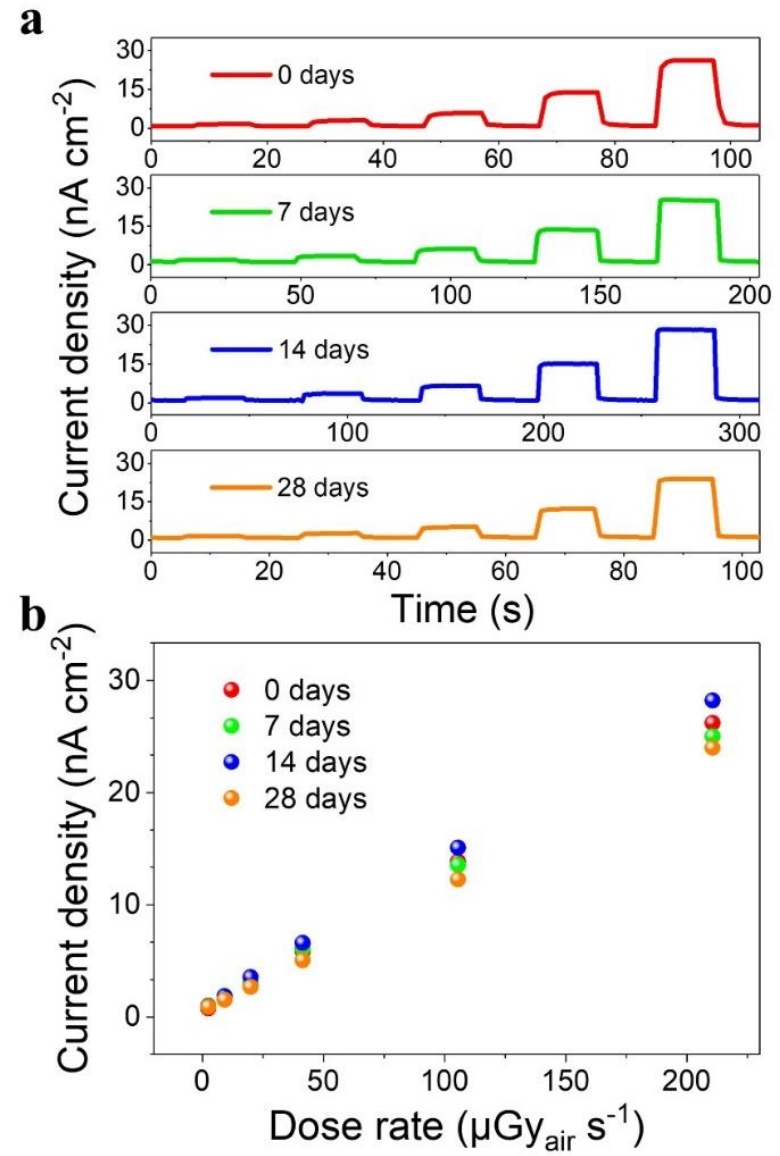

Figure S9. The long-term device stability of the optimized EPXDs encapsulated with a cover glass. 


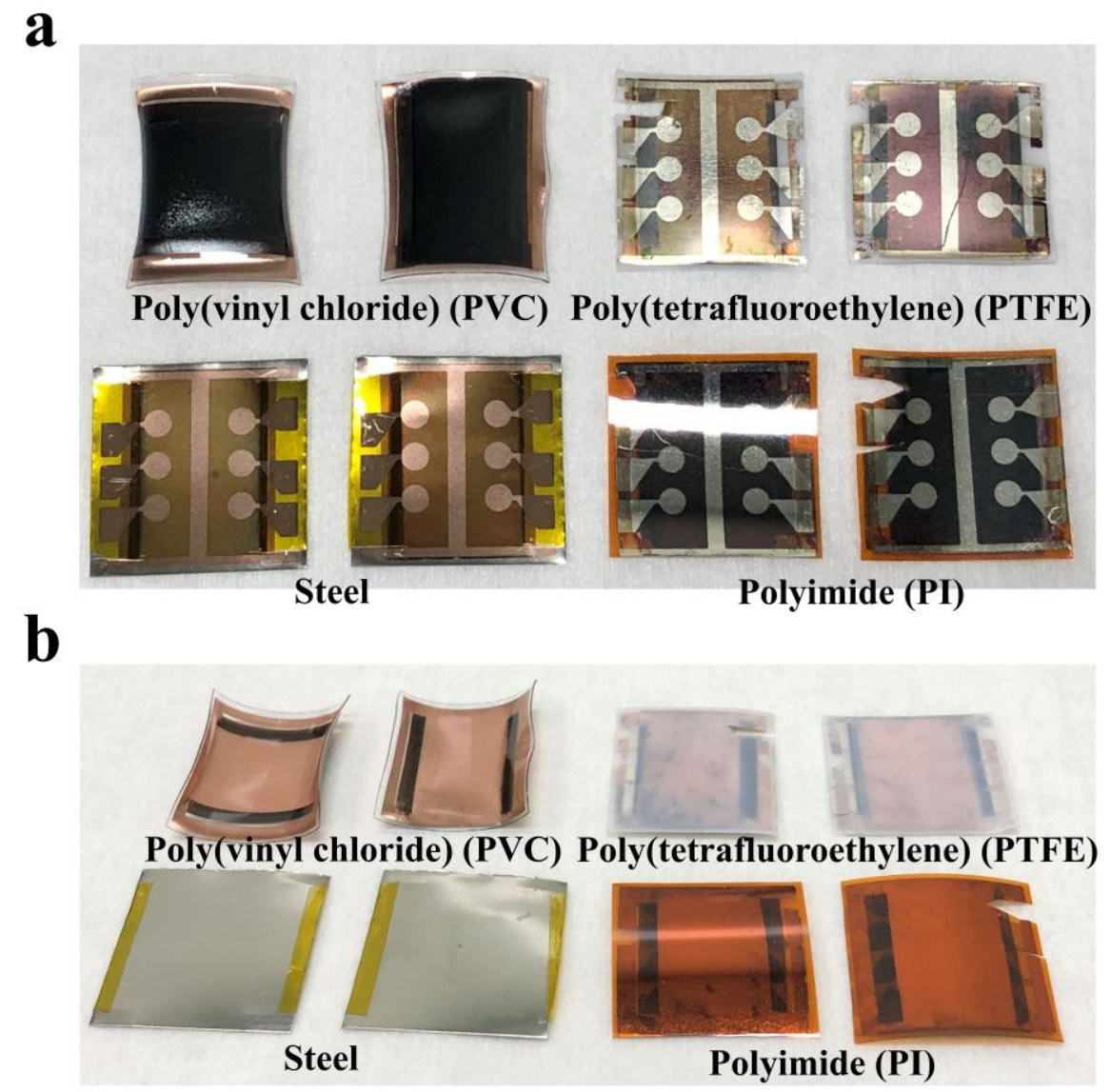

Figure S10. The comparison of the photographs of the (a) front and (b) back view of the EPXDs based on various flexible substrates.

a

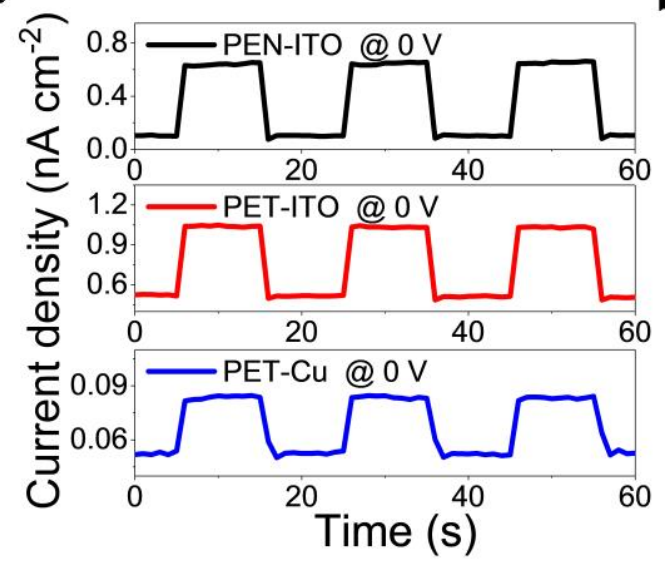

b

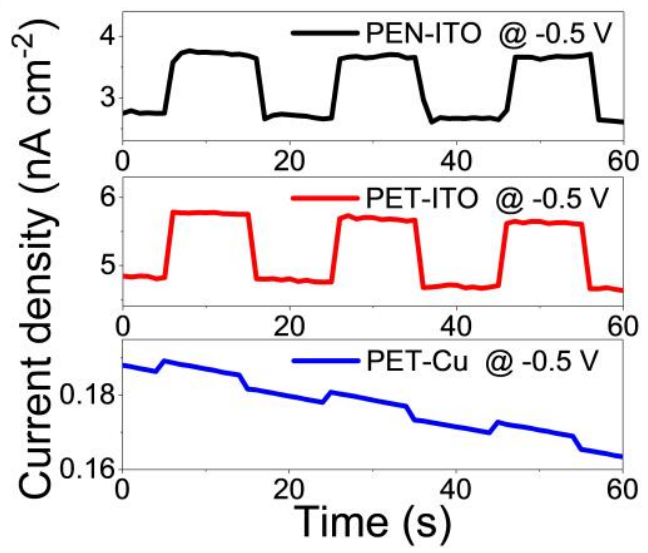

Figure S11. Repeated X-ray response switched between 2.5 and $105.6 \mu \mathrm{Gy}_{\text {air s}} \mathrm{s}^{-1}$ of flexible devices based on various flexible substrates with bias voltage of (a) $0 \mathrm{~V}$ and (b) $-0.5 \mathrm{~V}$. 